\title{
INTERNATIONAL PORTRAIT SERIES
}

No. 5

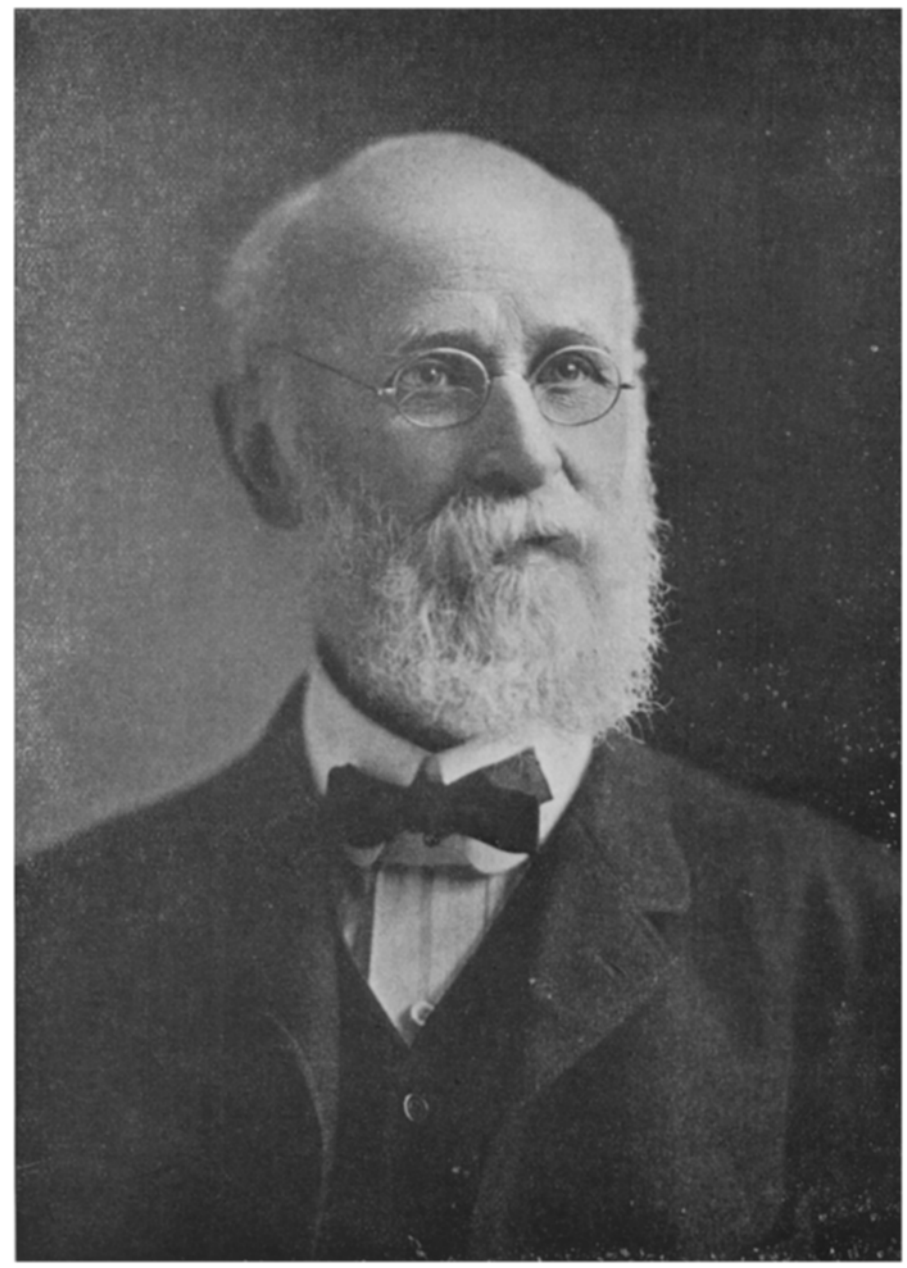

Green Vardiman Black, D.DiS., M.D., D.SC., LL.D. (1836-1915)

Principal Works:

The Formation of Poisons, 1884.

Periosteum and Peridental Membrane, 1887.

Descriptive Anatomy of the Human Teeth, 1889.

Operative Dentistry, 1908.

Special Dental Pathology, 1915. 


\title{
The International Journal of Orthodontia
}

Editor: Martin Dewey, D.D.S., M.D.

\section{ORIGINAL ARTICLES}

\section{A SYSTEM OF POSITIVE AND PAINLESS TOOTH MOVEMENT. *}

\author{
By Ray, D. Robinson, D.D.S., Los Angeles, CaL.
}

$\mathbf{W}$

ONDERFUL advances have been made in both the science and the art of orthodontia. Men of splendid mental capacity and wonderful technical ability have devoted their lives to its advancement, and where but a decade or two ago there was nothing of science, and but little of art, there now exists a splendid science and a beautiful art. The classification of malocclusion, the compiling of the knowledge of the causes of malocclusion, the advance in the knowledge of the surrounding tissues and the changes which take place in them incident to tooth movement, the improvement in types and designs of orthodontic appliances and the materials from which they are made are all worthy of our highest praise and commendation. The fame of the men who have done these several great works should be imperishable.

In offering criticism of the appliances now before the profession, your essayist wishes it distinctly understood that he is not criticizing the appliances, for their day, nor their creators at all. They have been splendid appliances in their time. Their development marked distinct steps in the evolution of orthodontic appliances, but it would be foolish to assume that, in them, perfection had been reached, or that it ever will be reached. It is only by analyzing the faults of these appliances, and comparing them with the one to be shown at this time, that we can arrive at any just conclusions regarding the value of the new type, as it will be found valuable, only if it overcomes faults found in the existing appliances.

Let the essayist then point out some of the faults of the present day appliances, as he sees them, and then consider the efforts he has made to overcome these faults.

First we will take up the older appliance of expansion arch and wire ligatures, as that has probably been more generally used than any other.

Objections.-First.-Any tooth movement secured by an appliance depending on the screw for development of force must be intermittent, and the force itself must rapidly vary in intensity, for a positive tooth movement 
is secured at the time the screw is tightened, and as the tooth moves the force rapidly lessens until in a short time no force is being exerted.

Second.-Any movement secured by the tightening of non-elastic ligatures connecting a tooth and a rigid, or semi-rigid, bar must be intermittent and again the force rapidly lessens until none is exerted. Movements of this character tend to produce soreness, unnecessary loss of bone tissue, and require longer periods of retention than those secured by an appliance in which the force is constant and uninterrupted over a long period of time. The abrasion of the tongue and lips by wire ligatures is a serious fault. Other objections are found in the use of ligatures of any kind in the injury done to the gums, and because they furnish a lodging place for accumulations of food between the teeth. Decalcification frequently results in these places. Again the ligatures become loose and displaced, either through design on the part of the patient, or through accident or stress of mastication, allowing a recession of the teeth, consequent soreness and a retarding of the progress of the work.

Third.-In the appliance depending on the screw, the force is developed at a point far distant from that to which it is to be applied, and must be transmitted through a long curved wire, which makes the application more uncertain than when the force is developed at, or near, the point where is to be applied.

Fourth.- The inability to lengthen any intermediate section of the arch often proves a very disagreeable feature, as, for instance, when the central and cuspid are lying in close approximation, or in contact, necessitating their being moved apart with but little labial movement; or in a similar condition in any curved part of the arch, where the teeth are to be carried along the line of occlusion, rather than labially. In such cases the movement mesially or distally without much labial movement is very difficult.

Fifth.-The type of appliance under discussion usually depends on one tooth in each lateral half of the jaw for its anchorage, and they must bear the full amount of force necessary to move all the anterior teeth, which is frequently sufficient to tax their support beyond the limit.

Sixth.-With this type of appliance, the teeth when moved must be tipped; their bodily movement is out of the question.

Seventh.- The constant surveillance which it is necessary to keep over this appliance, and the frequent applications of force necessary to keep the teeth moving are distinct disadvantages.

Eighth.- After a case has been completed the appliance must be removed and another constructed and adjusted in its place for retention. The construction of a proper retaining device of ten proves more difficult than the correction of the case itself. This is also a serious and unhappy time for the patient, as the construction of an appliance on teeth that have been moved, and are still loose, is usually a very painful operation.

Tube and Pin Appliance: Objections.-First.-The technic necessary properly to construct and apply this device is so severe and exacting as to place it out of the question, except in the hands of the most skillful technicians. Many of the men who have had their training in the construction 
and use of this appliance from the best authority, have given up its use, and others say they use it only occasionally.

Second.-The wire when small enough to serve properly for root control is not strong enough to expand properly a mature dental arch without the use of loops, for which there is no provision. Many are securing their expansion with the older form of arch and finishing with this type.

Third.--Root control is only feasible in a part of the arch. Those cases requiring great expansion, with consequent tipping of molars and bicuspids with the older appliances, are still being completed with the latter teeth badly inclined buccally.

Fourth.- The rotation of teeth requires either the use of rubber wedges or ligatures.

Fifth.-This appliance is subject to the same disadvantages of the abrupt application of force by the screw action as was the older form, but in a less degree.

Sixth.-If a band breaks or becomes uncemented, it is necessary to remove the whole arch before the band can be replaced.

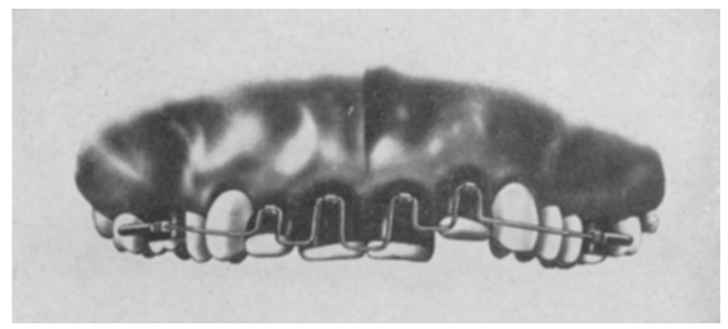

Fig. 1.

Your essayist is deeply sensible of the difficulty before him when he attempts to convince you that any appliance will meet and overcome all these objections, without itself developing other objections. To that end he will now show the appliance which he wishes to present, and its operation, after which he will take up the bad features of the other appliances as he has enumerated them, and we shall see if they have been met and overcome, or if he must be branded as an iconoclast-one who tears down and never builds up. After that it will be for you to say in your discussion whether the appliance, as he shows it, has other faults.

Except the force of gravity, there is probably no force in nature so constant in its operation as that of a coiled spring, as even the pressure of the atmosphere varies with the temperature, the elevation above or below sea level, etc. The coiled spring, in season and out, high or low, hot or cold, always works and never varies. Closely allied to the coiled spring is the looped spring. It has all the advantages of the coiled spring, and has the further advantage that it lies in a flat plane. It is this force which we are to consider today, with a new form of attachment to the teeth.

- With your permission, the essayist will digress long enough to explain some of the steps he has taken in developing the appliance. The appliance was first made with short, split, round tubing soldered to the labial 
surfaces of the incisor bands near the gingival line. The arch made of $.022^{\prime \prime}$ wire was threaded at its ends which were placed in tubes on the molar bands. The arch wire was engaged in the short split tubes, and at the points where it emerged, was bent sharply toward the incisal edge. It was allowed to extend nearly to the cutting edge, and was then bent at right angles and passed to the next tooth, when it was bent at right angles again toward the gingival line. When it reached the line of the split tubing it was bent again at right angles and passed through the next tubing and so on for each tooth (Fig. 1). By the proper bending of the wire, pressure could be brought to bear on the incisal ends of the teeth in the lingual direction, and at the same time it would pull labially on the gingival portion of the tooth. In this way the apeces of the roots were moved labially, and the incisal ends lingually. The appliance as described, did very good work in certain instances, but its usefulness was restricted to a very small percentage of cases. The things of greatest interest learned were the length of time such an appliance would continue its pressure, and the absence of all soreness.

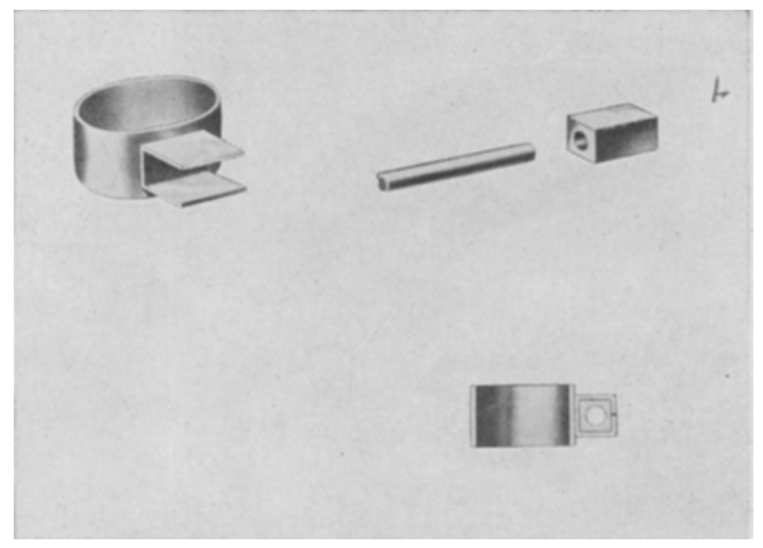

Fig. 2 .

After this, came the thought that better control could be secured if the rotation of the arch wire in the tubing could be prevented. To this end square split tubing was secured, and a square wire used, but a new difficulty immediately arose. When the wire was made into loops, it was found that no two loops had the same resistance, for a square wire bent in the direction of one side will not have the same resistance as though it is bent in the direction of a corner, and all the different angles between the flat side and the corner give different results. A triangular wire was then tried, then a flat wire with a groove on one side to provide means for locking into a suitable form milled from solid metal and soldered to the tooth bands, but they all presented difficulties. Then came the idea of squaring a round wire just at the points where it was to be locked to the bands. When a wire small enough to give just the right delicate resilience required for the work was squared, the squared portion was too small to handle, and when it was made large enough to handle, it was too stiff to give the results desired. After a period of study, came the idea of using hollow square blocks soldered on the arch 
wire at the places where the attachments were to be made (Fig. 2). That was the first real step in developing the present appliance. The ability to lock the arch wire itself into the seats without soldering on attachments was at first held by the essayist to be a necessity, but after once preparing an arch in this way, it developed that the technic is so simple as to bother no one. The possibility of making an error in alignment in soldering the block to the arch is practically nil, and the advantage gained is so great that there can be no question that the use of the round wire and the soldered blocks will be accepted as more desirable than the use of the angular wire.

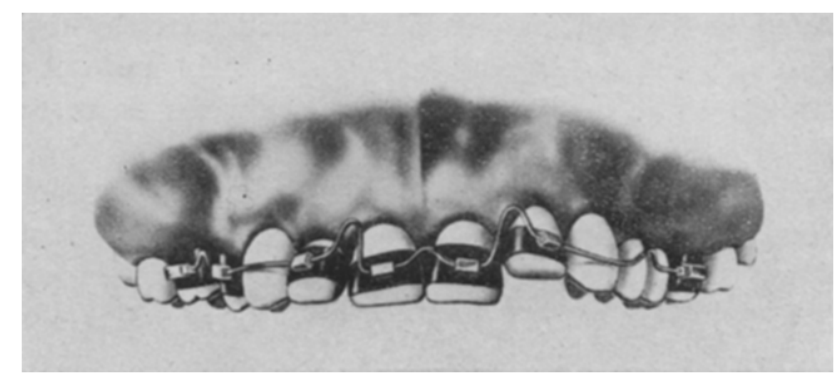

Fig. 3 .

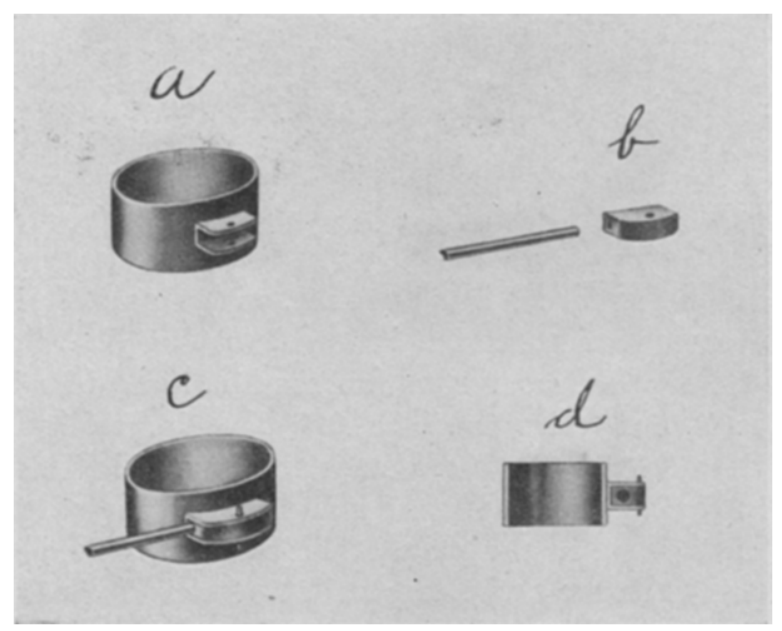

Fig. 4.

Iridio-platinum seats into which the blocks accurately fitted, were constructed and soldered to the tooth bands. When the blocks were soldered to the arch wire and locked into the seats by bending their edges, the attachment between the arch wire and tooth was rigid (Fig. 3). The appliance was first published in this form, and much good work was done with it as it was then used, but a serious fault developed. When the seat was made thin enough to bend over and lock the block in place, it was so frail that it of ten became loose under continued pressure and the stress of mastication, allowing the block and arch wire to slip, and thus the force was not applied as it should have been. Then began the hunt for a lock that would hold or release the wire as 
desired. No fewer then sixteen good, bad and worse forms of locking devices were tried and cast aside. Then came the fortunate day when the form now used was designed. It has been in use eight months. More than a hundred dozen have been used, and so far as can be learned, not one has allowed the wire to slip. Never have they failed to release when release was wanted.

The attachment between the tooth and the arch is made by an interlocking seat (Fig. 4a) and block (Fig. $4 b$ ), the former being soldered to the tooth band, and the latter to the arch wire. The seat consists of a flat oblong base and two flat parallel walls rising at right angles to the base. The walls are rounded at the top, and each is pierced near the top with a small hole, the two holes being in alignment. The seat is made of platinum-gold and is $.022^{\prime \prime}$ thick. The distance between the walls or across the base of the seat is $.040^{\prime \prime}$.

The block is of platinum-gold, but harder than the seat, and is $.040^{\prime \prime}$ thick and shaped to fit inside the walls of the seat above described, except that it is $.005^{\prime \prime}$ greater in elevation than the side walls of the seat, which pro-

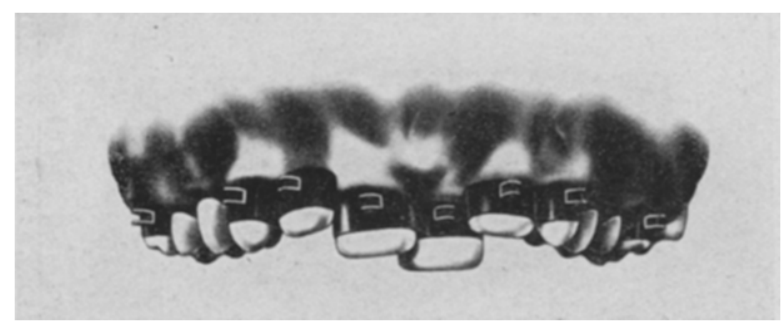

Fig. 5.

vides means for its being forced into the seat, with a pair of pliers, when there is stress on the arch wire to which it is soldered. The block (Fig. $4 b$ ) is pierced by two holes, the larger runs longitudinally and its bore is equal to the diameter of the arch wire used, or $.020^{\prime \prime}$. The smaller hole is through the transverse diameter of the block near the top and is in the exact place to be brought into alignment with the two holes in the seat walls when the block is forced into place in the seat. The block is locked into the seat by placing a delicate pin through the seat walls and the block (Fig. $4 d$ ). When so locked, no play is possible. The two parts are made to fit to the minutest fraction of an inch, and as they have three flat walls in apposition, and are locked firmly together, they do not permit of any play whatever. The molar and anterior attachments are alike, except that the molars are the longer (Fig. 4c.)

It is not to be supposed that the complete technic for all classes of cases can be given in an essay of this character. A general outline of the technic is all that can be attempted.

The technic of construction is very simple. The tooth bands are first constructed, and to their labial suirfaces are soldered the seats (Fig. 5). No necessity exists for getting them into alignment with each other, or of getting them at exactly right angles to the long axes of the teeth. If they be placed somewhere nearly at right angles it will be sufficient. After the bands with 
the seats attached have been cemented to the teeth, the arch wire is prepared as follows: A piece of arch wire is selected and enough blocks to correspond with the number of tooth bands are threaded on the wire; a molar block being first and last with the anterior blocks between. One of the molar blocks is now soldered to the wire. The block is next introduced into the seat in the mouth and the arch wire is bent in such a way as to bring it to lie through the next seat. If there is to be any change made in the relative position of the molar and the first tooth to be engaged, anterior to it, a loop should be made in the wire in such a position that it will lie near, without touching, the gum, and then bring the wire to lie through the next seat. When this has been done, bring forward the next block on the wire until it is approximately in the proper place to go into the seat. Next, with a pair of crimping pliers having a delicate projection in one beak, crimp the block on the wire; now the block may be forced into the seat and if it not in its proper place on the wire, it can be forced into place by grasping the ends of the seat and block in a pair of pliers and bringing force to bear. As they are exactly the same length, the block must go to its proper place. The arch wire is now removed from the mouth and the block soldered, there being

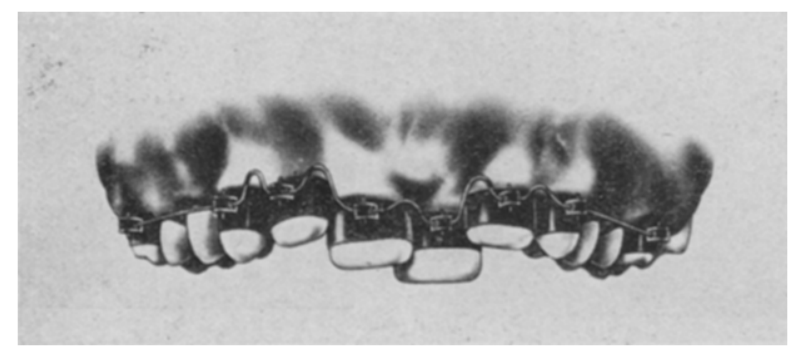

Fig. 6.

little danger of displacing the block on the wire because after crimping the block, force must be used to change its position on the wire. Each block is, in its turn, brought to its proper place and soldered in a like manner, loeps being formed wherever necessary. After the last block has been soldered, any remaining portion of the wire is cut off. It will now be found that when the arch is placed in position each block will lie passively in its seat (Fig 6).

When the work required of the arch wire has been determined it is bent into the necessary form and is then replaced in the mouth and each block is forced into its corresponding seat and locked there. If an effort is to be made to bring all the teeth into harmony with the line of occlusion with one application of force, measurements of the incisors and cuspids will be made, and by using the Hawley arch predetermining transparencies the true arch for the individual case will be determined. Guessing is eliminated with the diagram of the true arch at hand. The loops in the expansion arch already constructed will be opened, closed or twisted, as may be required, to bring the wire arch to the size and shape of the ideal dental arch determined on, with each block occupying the position in the ideal arch that the labial or buccal side of the tooth should occupy. After this has been done, the arch wire is placed in the mouth and each block in its corresponding 
seat. If we could depend on a wire developing onehundred per cent of resilience, nothing beyond this would ever be necessary, but that of course we cannot do. It will be observed that the attachment between the tooth and the wire arch is rigid. There is no chance for any play between them. Any force brought to bear through the arch wire must, in time, be registered in the tooth, and, as the attachment is rigid, the tooth must move as a whole, the root as well as the crown. In fact the control over the tooth is absolute. It will be argued that so small a wire will not produce enough force to move the teeth in a mature jaw, or expand an arch. The answer to that argument is that it does not require much force to do anything necessary in the moving of teeth, if that force be continuous over a long period of time. The arch as described is a looped spring and the essayist will show you proof that it will

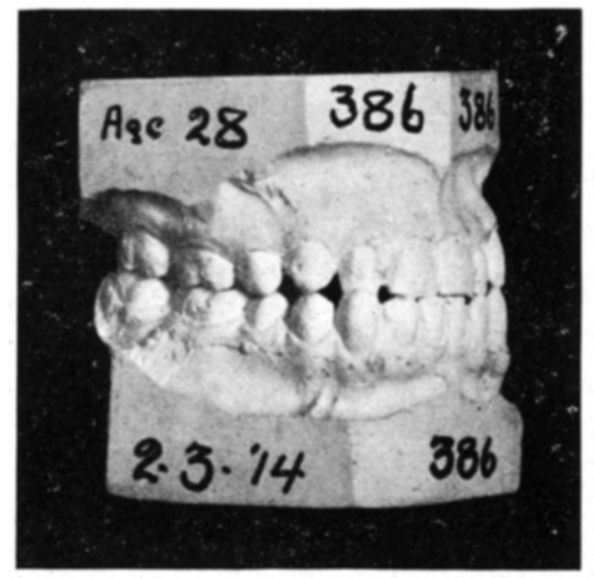

Fig. 7.

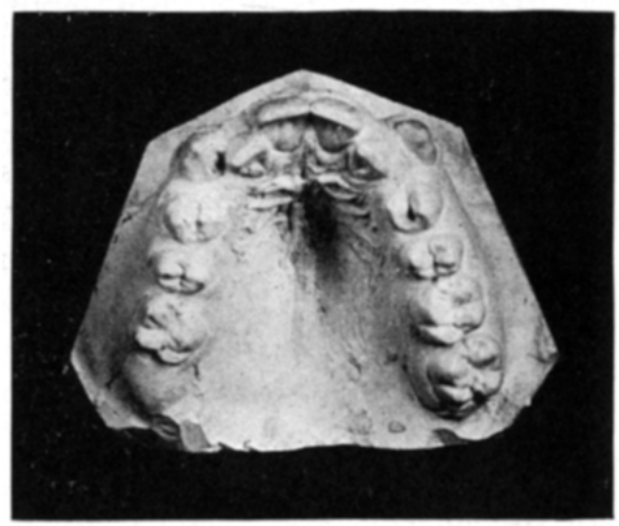

Fig. 9.

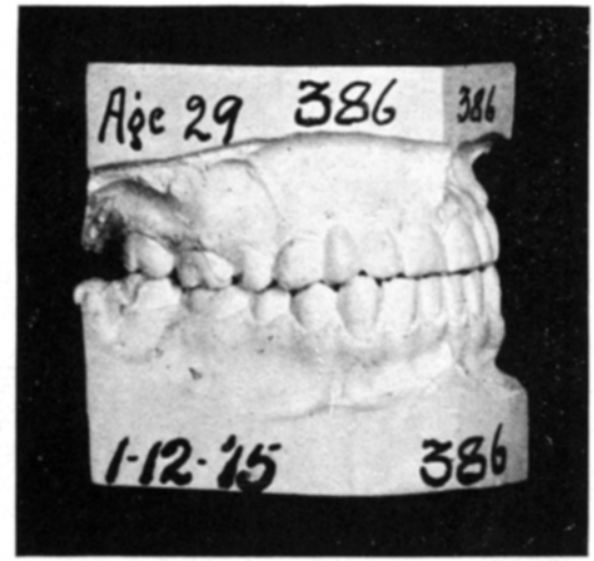

Fig. 8.

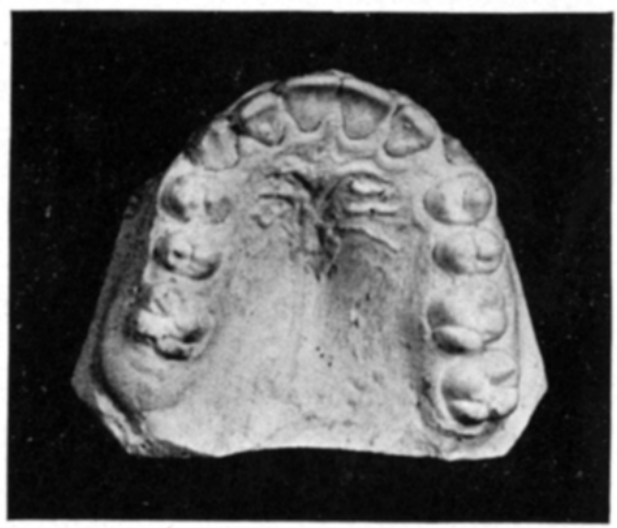

Fig. 10.

continue its pressure over a period of four months without tightening, and that it will expand an arch, and that it will move teeth in an adult jaw, and move them so gently, slowly and steadily as not to produce even the slightest soreness, and that when they are so moved they require but a minimum of retention.

Model shown in Fig. 7 is that of a lady of twenty-eight, who had a cuspid 
extracted when she was fifteen years of age, allowing the lateral and first bicuspid to come into actual contact with a decided lingual movement of all the superior incisors. The appliance was adjusted on February 10, 1914. It was tightened on March 3, April 7, and May 5. The teeth continued to move until the latter part of June, after which nothing was done until September 8 , when certain events in the lady's career made it highly desirable that the appliances be removed. This was done, and a bridge was made by her dentist to supply the missing cuspid. The model shown in Fig. 8 was made on January 12,1915, more than four months after retention was

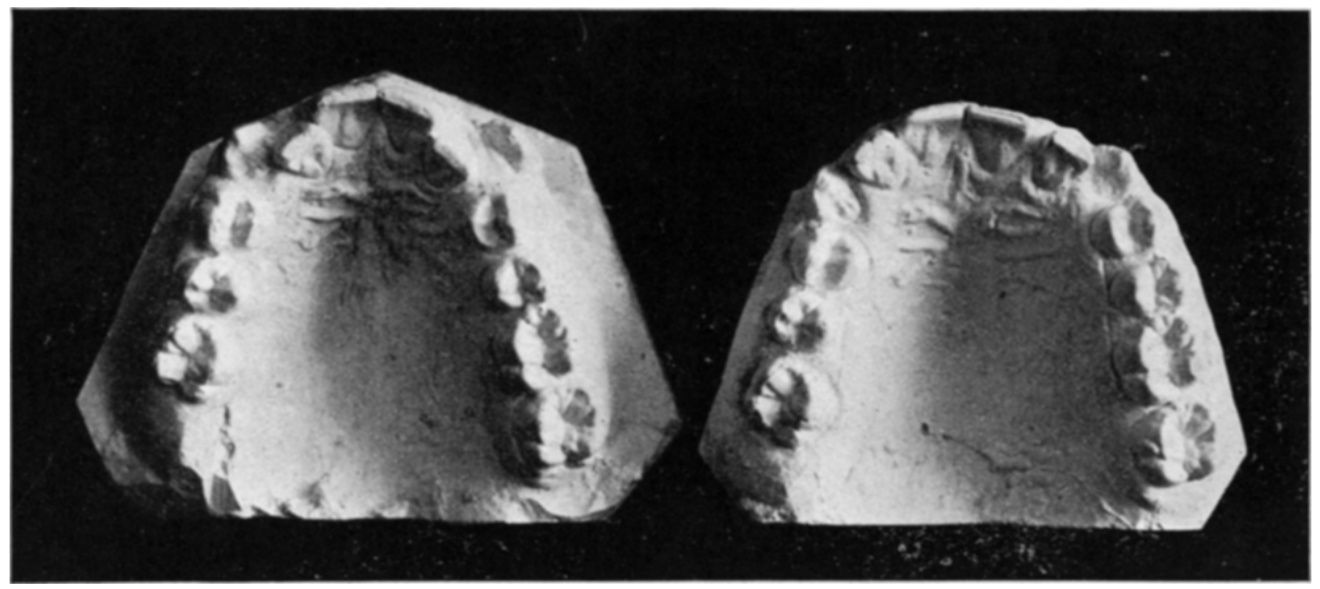

Fig. 11.

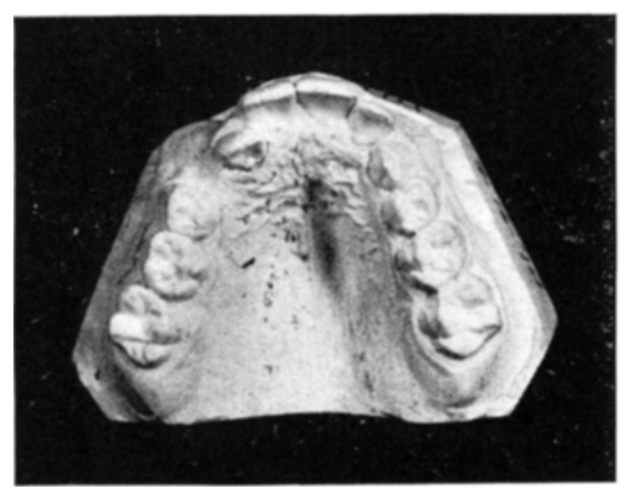

Fig. 12.

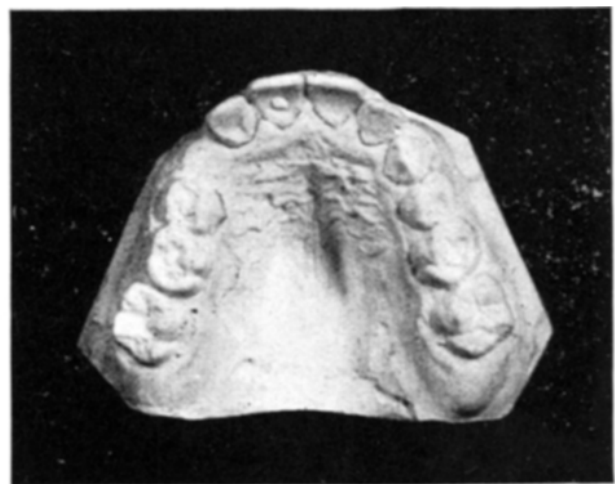

Fig. 13.

removed. The occlusion originally was such that the incisors had been much worn, and when they were placed in their proper places there was no overbite. The retention period was less than three months, and yet it will be observed that only one tooth, the right central incisor, has shown any movement, and it but the very slightest.

The case was selected for illustration on account of the advanced age of the patient and the difficulty usually attending the retention of such a case at that age.

Fig. 9 shows a case of a boy of fourteen. The model was made on June 11,1914 , and the upper appliance adjusted on June 18. The writer's absence 
from the city followed by the patient's absence prevented even an inspection of the case until October 14 . The change was so startling that the appliance was removed and a model was made, which is shown in Fig. 10. It will be observed that there has been but little expansion in the molar region, and that there is not enough room for the cuspids, but it will also be observed that there has been a great amount of movement and that all of the movement of each tooth has been in the desired direction. Fig. 11 shows what the second application of force accomplished.

Fig. 12 shows a case of a boy of eleven. The model was made on September 16. The upper appliance was adjusted on September 30. Permission to allow the case to go three months without further attention was asked. Nothing was done until December 16, at which time the model shown in Fig. 13 was made.

The work shown was all done with a platinum-gold wire. Your essayist at a meeting of the American Society of Orthodontists last year said that, if he could get a steel wire with a noble metal sheath so that he could solder

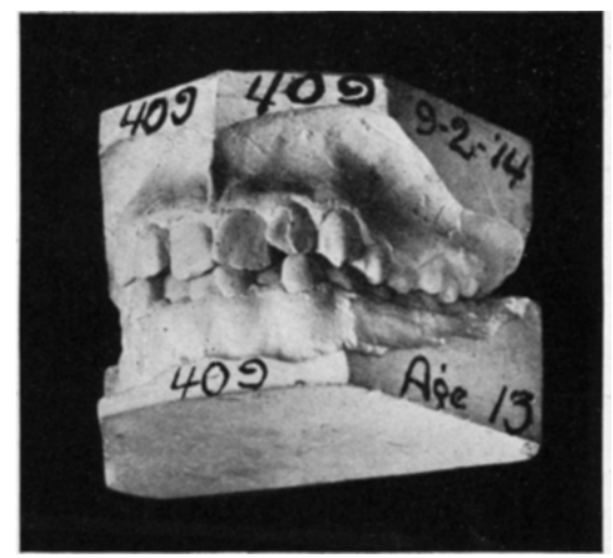

Fig. 14.

to it, and could then find a proper means for tempering it, he could finish sixty per cent of Class I cases with not more than two applications of force. In answer to his prayer for wire with better resilience, listen to this quotation from the report of Dr. Weston A. Price, chairman of the Research Committee of the National Dental Association:

"Tungsten with its elastic content about twice that of steel, its melting point nearly twice that of platinum, its stiffness about six times that of thirty per cent iridium in platinum and with the remarkable property that it does not anneal or lose its stiffness and elasticity even when heated to the melting point of gold, makes it particularly adaptable for many parts of orthodontia appliances. After testing tungsten in practical use in expansion arches for about a year, and after extreme laboratory tests, we believe it to be superior for various forms of expansion arch to any metal that we have heretofore found for the following reasons: The required elasticity and rigidity can be secured with smaller sizes. Attachments of any kind may be made to it with pure gold or with any karat of gold solder, without changing its stiffness at the point of attachment. The entire appliance and its con- 
necting parts can be of such metals that not only is there practically no discoloration or oxidation of the appliance, but also with the very slight electrolytic potential difference between the various parts, thereby reducing the electrolysis.

"This great elasticity of tungsten makes it particularly desirable and adaptable for the new type of expansion arches, a much smaller size of

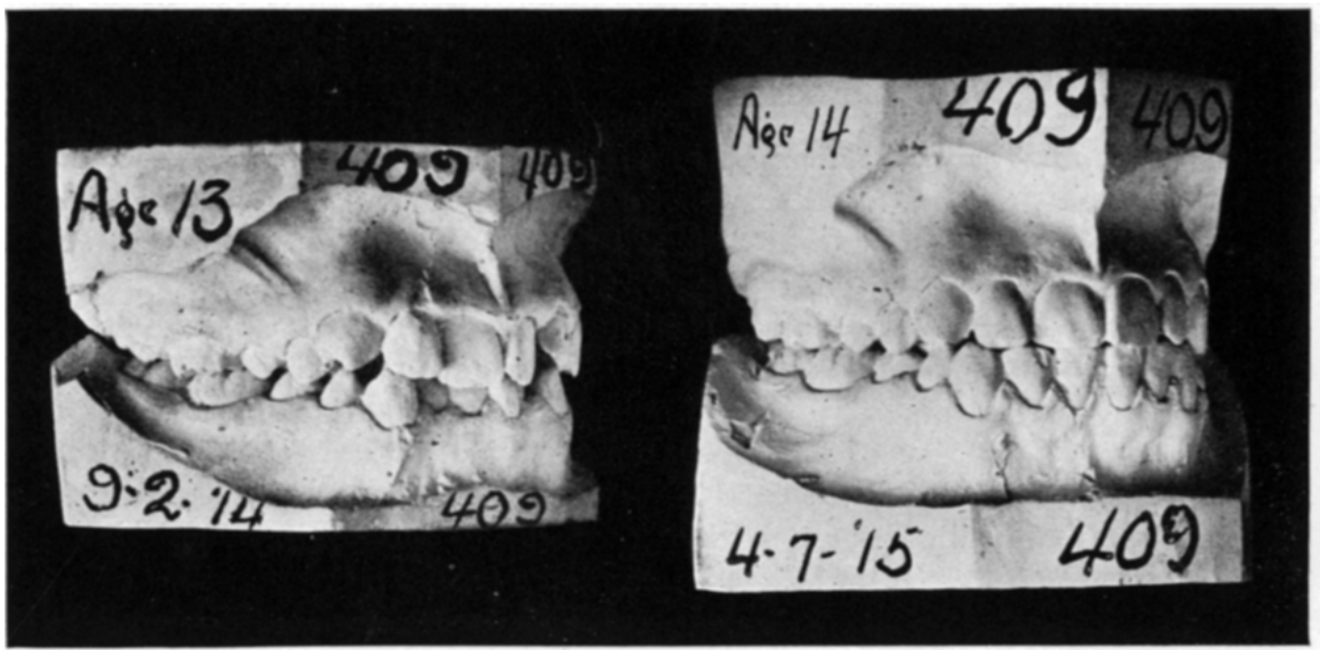

Fig. 15.

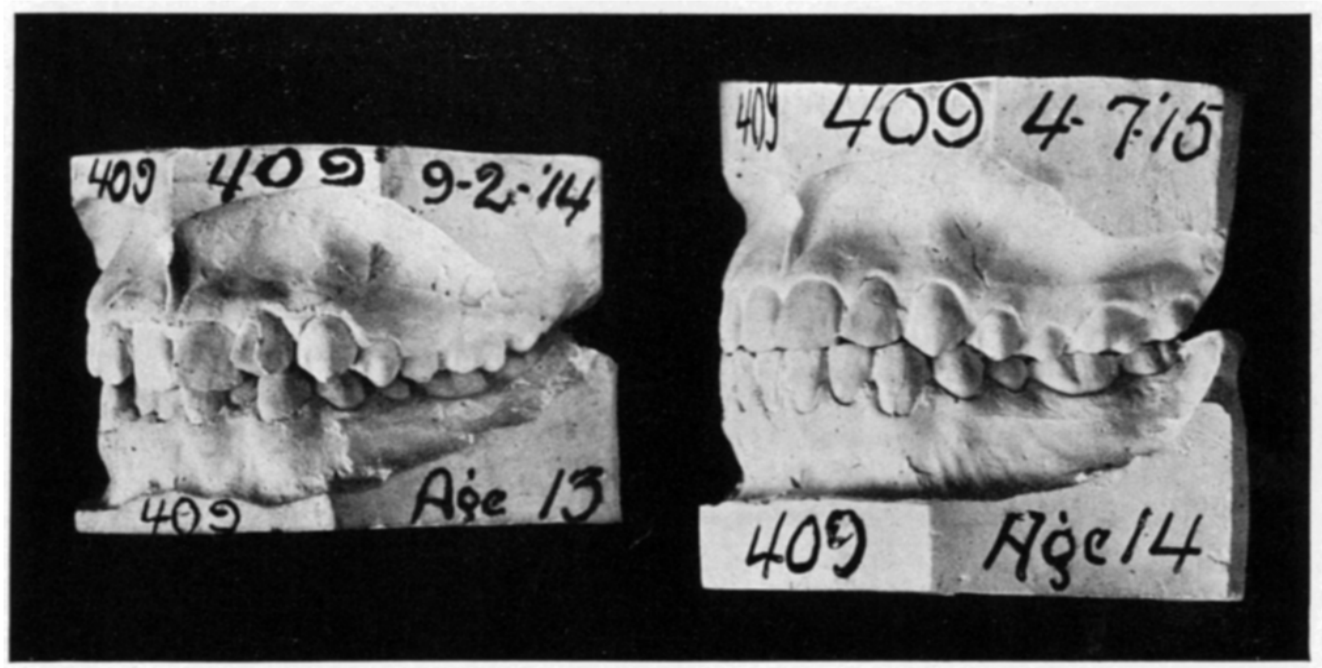

Fig. 16.

tungsten can be used for this purpose than may be in the other metals and still have a large factor of stiffness for expanding the arch. The attachments can be made with any gold solder without danger of sof tening or annealing the arch and the bends can later be taken out cold, if not sharp, and if sharp, by heating to a dull red while bending. It does not crystallize and break like gold clasp bar, and similar metals, under the stress of mastication." 
With such a wire as Dr. Price describes it is possible, in a large percentage of cases, after drawing a diagram of the ideal arch for each case with the aid of the Hawley arch predetermining transparencies, to construct a wire arch which will, when properly locked to the teeth, bring them into harmony with the line of occlusion without further attention. In another large percentage of cases it will require two applications of force. The first to secure the proper width to arch, before attempting the placing of individual teeth, such as rotation, etc., and the second to secure such placing and rotation. In others it will require more than the two, but your essayist readily believes that there is no case, of suitable age if it is amenable to any treatment, which cannot have the best, that is possible for the case, completed in a half-dozen applications of force, and that without pain.

In August, 1914, there was placed in your essayist's hands a piece of tungsten wire with a gold-palladium surface, such as has been produced by the Research Committee of the National Dental Association. The case shown in Fig. 14 was selected as suitable for a trial of this wire. Very careful and exacting measurements of the teeth were made and the true arch was determined to the best of the operator's ability. The attachments were made and cemented to the teeth. The wire arches were then constructed as described above. These were both attached to the teeth, as given in the technic, on September 2, 1914. The case was seen on the average of once a week, but only for observation, as nothing was done until the appliance was removed on April 9, 1915, at which time the work had developed to the point shown in Figs. 15 and 16 . Your essayist makes no claim that normal occlusion was then secured, but leaves to your own judgment the value of an appliance which will perform the work shown in these two illustrations without attention and without even tooth soreness.

Your essayist wishes it distinctly understood that in ordinary handling of a case, he would not permit it to go so long a period without attention. His object in doing so in this case was to allow the appliance to work to the limit of its ability and in so doing test the value of the new tungsten wire.

Let us now see if this appliance has overcome the objections which its creator has raised to the previously existing appliances, and if it has overcome them, let us see if it has developed any new faults. If it has overcome these objections and has not developed a new lot of troubles peculiar to itself, it is worthy of your attention-otherwise, it should be discarded.

To state the difference in the operation and the principles of force employed by the different appliances in a few sentences and bring these differences sharply before you so that there may be no confusion in your minds, let it be said: The older Angle appliance uses a rigid arch formed in the shape of an ideal dental arch and depends on the use of ligatures to bring the teeth to this form. This is supplemented by the ability to lengthen the arch by a screw at either of its ends.

The tube and post alliance uses a semi-rigid arch bent to the form the teeth occupy in malocclusion and depends on the force produced when, at intervals, the arch is removed from the mouth and bent into a shape more nearly approaching the ideal dental arch. This force is supplemented also by the ability to lengthen the arch by a screw at either of its ends and by the use of wedges for rotation. 
The appliance presented here depends on a non-rigid arch formed into the shape of the ideal dental arch, which is so flexible as to permit of its being bent into the form of the dental arch, with the teeth in malocclusion, and so resilient, by virtue of its composition and the loops into which it is formed, that it will return to its original form of an ideal dental arch and carry the teeth with it. The greatest feature of the appliance is that by virtue of the loops into which the wire is bent, it is capable of exerting force over a long period of time, and is capable of moving a tooth relatively great distance without being tightened, or at any time exerting more than a very little force.

The objections to the older Angle appliance were: First, to the intermittence of the force developed by the screw. That has undoubtedly been overcome.

The second was to ligatures, there being many reasons why they are objectionable. This appliance uses no ligature. The third was to the fact that the force was developed at a point distant from the one where it was applied, and was not always reliable for that reason. In this appliance the force is developed at the point where it is used.

The fourth was to the inability to lengthen any intermediate section of the arch. That has certainly been overcome. The fifth was to faulty anchorage. In this appliance reciprocal anchorage is carried to the $n$th degree. The sixth was to the inability to secure bodily movement of teeth. With this appliance there is perfect control over all the teeth.

The seventh was to the constant surveillance and attention which the appliance required. That this appliance needs little of either is quite evident. The eighth was to the necessity of making retaining devices, and the length of time it was necessary to wear them. This appliance is its own best retainer, and the retention period is much reduced.

The objections to the tube and post appliance were: First, to the severity of the technic necessary. The necessary technic for constructing this appliance is simple and is along lines easy to follow.

The second was to the lack of provision for proper expansion. That has been overcome in this appliance, and with a wire but two-thirds the diameter of the one previously used.

The third was to lack of root control in the molars and bicuspids. This appliance gives control over all the teeth. The fourth was to the lack of ability to rotate teeth without wedges or ligatures. That surely has been overcome.

The fifth was to the abrupt application of force by the screw. That too has been met and overcome. The sixth was to the difficulty of repair. In this appliance any band can be removed and replaced without disturbing any other part of the appliance.

The question now arises: Does the appliance as described have any faults, and the answer is yes. The lack of soreness has, strange to say, proved a bad feature, for the reason that when there is no soreness, the patient is very apt to put too much strain on so delicate an appliance by biting into apples, hard toast and other substances, and frequent breakage of the arch wire has resulted, but the new tungsten wire has overcome even this. 